\title{
Large-offset P-wave traveltime in layered transversely isotropic media*
}

\author{
Mohammad Mahdi Abedi ${ }^{1}$, David Pardo ${ }^{2}$ \\ IBasque Center for Applied Mathematics, Bilbao, Spain. E-mail: mabedi@bcamath.org (corresponding \\ author). \\ ${ }^{2}$ University of the Basque Country, Department of Applied Mathematics, Statistics, and Operational \\ Research, Spain; and Basque Center for Applied Mathematics, Bilbao, Spain; and Ikerbasque, Bilbao, Spain. \\ E-mail: david.pardo@ehu.es
}

\begin{abstract}
Large-offset seismic data processing, imaging, and velocity estimation require an accurate traveltime approximation over a wide range of offsets. In layered transversely isotropic media with vertical symmetry axis (VTI), the accuracy of traditional traveltime approximations is limited to near offsets. Herein, we propose a new traveltime approximation that maintains the accuracy of the classical equations around zero offset, and exhibits the correct curvilinear asymptote at infinitely large offsets. Our approximation is based on the conventional acoustic assumption. Its equation incorporates six parameters. To define them, we use the Taylor series expansion of the exact traveltime around zero offset, and a new asymptotic series for infinite offset. Our asymptotic equation shows that the traveltime behavior at infinitely large offsets is dominated by the properties of the layer with the maximum horizontal velocity in the sequence. The parameters of our approximation depend on: the effective zero offset traveltime, the normal moveout velocity, the anellipticity, a new large-offset heterogeneity parameter, and the properties of the layer with the maximum horizontal velocity in the sequence. We apply our traveltime approximation: (1) to directly calculate traveltime and ray parameter at given offsets, as analytical forward modeling; and (2) to estimate the first four of the aforementioned parameters for the layers beneath a known high-velocity layer. Our large-offset heterogeneity parameter includes the layering effect on the reflections traveltime.
\end{abstract}

Keywords: traveltime, layered, VTI, high-velocity layer, modeling.

\footnotetext{
* This paper is published in GEOPHYSICS, VOL. 86, NO. 3 (MAY-JUNE 2021); P. C65-C74, 10.1190/GEO2020-0415.1
} 


\section{INTRODUCTION}

Modern high-dynamic-range acquisition systems that record seismic data over extended geometries have enabled high-fold wide-offset studies in exploration seismology (Colombo, 2005). In transversely isotropic with vertical symmetry axis (VTI) media, the larger the offset range, the wider the angle coverage and the more noticeable the anisotropy effect. This brings the opportunity for a more accurate estimation of the anisotropy parameters, but also requires suitable algorithms for accurate data processing and imaging.

An essential part of many seismic data processing (e.g., Abedi et al., 2019a, 2019b), imaging (e.g., Dai and Li, 2001), and velocity and anisotropy estimation (e.g., Alkhalifah, 1997) algorithms is an approximation of the traveltime-offset equation. Existing popular traveltime approximations are proposed for basic models, such as a homogenous anisotropic (Muir and Dellinger, 1985; Alkhalifah and Tsvankin, 1995; Fomel, 2004; Alkhalifah, 2011; Abedi and Stovas, 2019a; Abedi, 2020), or an inhomogeneous isotropic model (de Bazelaire, 1988; Causse et al., 2000; Taner et al., 2005). However, real earth models are both inhomogeneous and anisotropic. Therefore, we commonly use the Dix-type (Dix, 1955) effective velocity and anellipticity (or heterogeneity) parameters suggested by Alkhalifah (1997) in the aforementioned approximations. Effective parameters are an average of the properties of the layers, calculated from a weighted average of the series expansion of vertical slowness around zero horizontal slowness. Using the effective parameters in a traveltime approximation ensures the traveltime accuracy only at near offsets.

Using one of the aforementioned traditional traveltime approximations to estimate the velocity and anellipticity parameters from wide-offset data, the results significantly differ from the theoretical zero offset effective parameters. Trying to convert the estimated parameters into the interval counterparts (the properties of each layer in layered models) by the Dix-type differentiation process (Alkhalifah and Tsvankin, 1995) results in augmented errors.

Few traveltime approximations are designed for vertically inhomogeneous anisotropic models, such as the methods of Ravve and Koren (2017), and Farra and Pšenčík (2020). Both methods عسث one ray at a given ray parameter or finite offset. Other traveltime approximations that use properties of one traced ray to accurately estimate parameters of their traveltime approximations in layered anisotropic models are the generalized moveout approximation (Fomel and Stovas, 2010; Stovas and Fomel, 2017) and its extended version (Abedi and Stovas, 2019b). These methods that use ray-tracing or the method of Ibanez-Jacome et al. (2014) that use effective isotropic velocities are suitable for forward-modeling but unfit for conventional velocity and anisotropy parameter estimation. The Farra and Pšenčík (2020) method is also based on the weak anisotropy assumption (that assumes the anisotropy parameters are close to zero, and the phase and group velocities are equal), which is less accurate than the acoustic assumption (Alkhalifah, 1998).

In this study, we first analyze reflections traveltime at infinitely large offsets by calculating the asymptotic series of traveltime squared in layered acoustic VTI media - at infinity- and obtaining a new effective parameter that is related to heterogeneity. Next, we propose a new traveltime approximation for these media that satisfies several terms of the Taylor series of traveltime squared at zero offset and the new asymptotic series at infinite offsets. We apply our approximation to explicitly calculate the traveltimes and ray parameters of reflections at given offsets (the forward problem), and estimate model parameters from reflections beneath a known high-velocity layer (the inverse problem).

\section{THEORY}

In multilayer VTI media, traveltime approximations conventionally use effective velocity and anellipticity (or heterogeneity) parameters (Alkhalifah, 1997). The effective parameters arise from different orders of traveltime derivatives at zero offset. These derivatives appear in different terms of the Taylor-series 
expansion of traveltime squared as (Hake et al., 1984; Tsvankin and Thomsen, 1994; Ursin and Stovas, 2006),

$T^{2}(X \rightarrow 0) \approx T_{0}^{2}+\frac{1}{V_{n}^{2}} X^{2}+\frac{1-S_{2}}{4 T_{0}^{2} V_{n}^{4}} X^{4}+\ldots$

where $T$ is traveltime, $X$ is offset, $T_{0}, V_{n}$, and $S_{2}$ are the effective zero offset traveltime, normal moveout (NMO) velocity, and second-order heterogeneity parameter, which is related to the effective anellipticity parameter $\eta_{e}=\left(S_{2}-1\right) / 8$ (Ursin and Stovas, 2006). Over a stack of $k$ VTI layers, these quantities are defined as (Alkhalifah 1997; Ursin and Stovas, 2006):

$T_{0}=\sum_{i=1}^{k} t_{0(i)}$,
$V_{n}^{2}=T_{0}^{-1} \sum_{i=1}^{k} t_{0(i)} v_{n(i)}^{2}$,
$S_{2}=T_{0}^{-1} V_{n}^{-4} \sum_{i=1}^{k} t_{0(i)} v_{n(i)}^{4}\left(1+8 \eta_{(i)}\right)$,

where $t_{0}, v_{n}$, and $\eta$ are zero offset traveltime, NMO velocity, and anellipticity parameter of each layer. We use small letters for parameters of each layer and capital letters for effective parameters. We have $t_{0}=2 z / v_{P 0}, v_{n}=v_{P 0} \sqrt{1+2 \delta}$, and $\eta=(\varepsilon-\delta) /(1+2 \delta)$, where $v_{P 0}$ is the vertical P-wave velocity, $\varepsilon$ and $\delta$ are the Thomsen (1986) anisotropy parameters, and $z$ is the thickness of each layer. The effective parameters in equation 2 are defined at zero offset.

For large-offset data, we calculate the asymptotic series of traveltime squared around infinite offset. We use the parametric equations of traveltime and offset as a function of ray parameter (equation A-1) and use the ray that directs to infinite offset. The parametric equation of offset tends to infinity when the ray parameter approaches the inverse of maximum horizontal velocity in the sequence of layers. We calculate the new asymptotic series following the method presented in Appendix A to obtain:

$T^{2}(X \rightarrow \infty) \approx \frac{1}{v_{h M}^{2}} X^{2}+\frac{2 t_{0 M}}{v_{h M}} S_{\infty} X+t_{0 M}^{2}\left(1+2 \eta_{M}+S_{\infty}^{2}\right)+\ldots$,

where $v_{h}$ is the horizontal velocity $\left(v_{h}=v_{n} \sqrt{1+2 \eta}\right)$, and the subscript $M$ denotes the properties of the layer with the maximum horizontal velocity in the sequence $\left(t_{0 M}, v_{h M}, \eta_{M}\right)$. Following Appendix A, the term $S_{\infty}$ is a part of the calculated series at infinity. It is a cumulative parameter over a stack of $k$ VTI layers and depends on the properties of all layers,

$$
S_{\infty}=\sum_{i=1}^{k} \sqrt{\frac{t_{0(i)}^{2}\left(v_{h M}^{2}-v_{h(i)}^{2}\right)}{t_{0 M}^{2}\left(v_{h M}^{2}-v_{h(i)}^{2}+v_{n(i)}^{2}\right)}} .
$$


The parameter $S_{\infty}$ is dimensionless, zero in a homogeneous medium (since there is only one layer with $\left.v_{h M}=v_{h(1)}\right)$, and positive in layered media; therefore, it can be introduced as a large-offset heterogeneity parameter.

The first three terms of the series in equation 3 form the curvilinear asymptote of traveltime-squared at infinitely large offsets, which is a shifted hyperbola. In equation 3 , the coefficients define the curvature, shift, and intercept of the shifted hyperbolic asymptote, respectively.

Figure 1 shows the square root of the series expansions in equations 1 and 3 in a layered VTI model. This figure shows the accuracy effect of adding each term into the series

\section{Traveltime approximation}

The generalized moveout approximation (Fomel and Stovas, 2010) and its extended version (Abedi and Stovas, 2019b) are flexible equations that produce highly accurate traveltime approximations in different media. These traveltime approximations have explicit parameter definition based on model properties for a homogeneous VTI media, certain vertically heterogeneous isotropic media, and homogenous models with a curved reflector (in the original papers). However, in layered models, they are unable to accurately reproduce the asymptotic traveltime behavior at infinitely large offsets (see Appendix B). Here, we modify the extended generalized moveout approximation (Abedi and Stovas, 2019b) functional form so that its odd-order derivatives do not vanish, and its asymptotic series at infinity has the same powers of offset as the exact series (Appendix B). The proposed approximation is given by

$$
T^{2}(X) \simeq T_{0}^{2}+\frac{X^{2}}{V_{n}^{2}}+\frac{A X^{4}}{V_{n}^{4}\left(\sqrt{T_{0}^{4}+2 B X^{2}+C X^{4}}+\sqrt{T_{0}^{4}+D X^{2}}\right)} .
$$

Equation 5 has six parameters $\left(T_{0}, V_{n}, A, B, C, D\right)$; in the following, we define them by matching the series expansion of equation 5 to the exact series around zero (matching equations 1 and B-3) and infinite offsets (matching equations 3 and B-10). At zero offset, we define $T_{0}$ and $V_{n}$ as in equation 2, and

$$
A=\left(1-S_{2}\right) / 2=-4 \eta_{e}
$$

At infinite offset, if $A /\left(v_{h M}^{2}-V_{n}^{2}\right)<0$, we obtain,

$$
\begin{aligned}
B & =A^{2} v_{h M}^{6} \frac{4 S_{\infty}^{2} V_{n}^{2}+\left(v_{h M}^{2}-V_{n}^{2}\right)\left(t_{0 M}^{2}\left(1+S_{\infty}^{2}+2 \eta_{M}\right)-T_{0}^{2}\right)}{V_{n}^{2}\left(v_{h M}^{2}-V_{n}^{2}\right)^{4}} \\
C & =\frac{A^{2} v_{h M}^{4}}{V_{n}^{4}\left(v_{h M}^{2}-V_{n}^{2}\right)^{2}}, \\
D & =4 A^{2} \frac{S_{\infty}^{2} t_{0 M}^{2} v_{h M}^{6}}{\left(v_{h M}^{2}-V_{n}^{2}\right)^{4}} .
\end{aligned}
$$

Therefore, equation 5 fits the exact traveltime in layered VTI media, via three constraints at zero offset and three constraints at infinite offset (the shifted hyperbolic asymptote). 


\section{Comments}

1. We obtain equation 7 under the condition $A /\left(v_{h M}^{2}-V_{n}^{2}\right)<0$. This condition arises from equating the first term of equation 3 to the first term of equation B-10 and solving for $C$. This condition is always satisfied if $\eta$ values in all layers above the reflector have the same sign. To ensure this condition is satisfied for all models, we propose the following definition for $A$,

$$
A=-\left|\frac{1-S_{2}}{2 v_{h M}-2 V_{n}}\right|\left(v_{h M}-V_{n}\right) \text {, }
$$

where $\mid$ is the absolute value operator. Equation 8 ensures the fitting constraints at infinity, but relaxes the third fitting condition at zero offset in models that the above mentioned condition is not met.

2. If at any offset $B<-\frac{T_{0}^{4}+C X^{4}}{2 X^{2}}$, we replace it with $B=0$. This eliminates the third constraint at infinity but keeps the approximated traveltime being real valued.

\section{NUMERICAL ANALYSIS}

We assess the accuracy of the proposed traveltime approximation in different multilayer VTI models. First, we use the fourth reflection in the earth model described in Table 1. For comparison, we use the approximation of Ravve and Koren (2017; represented in equation B-2). To compare with a traditional approximation, we use the Alkhalifah and Tsvankin (1995) approximation (originally proposed for a homogeneous VTI layer), with the conventional effective velocity and anellipticity (Alkhalifah, 1997) as its parameters. In Appendix B, we also modify the parameter definition for the Tsvankin and Thomsen (1994), to have one fitting constraint at infinity (equation B-11). Figure 2 shows the relative error $(R E)$ of each traveltime approximation as,

$R E=100\left|\frac{T_{\text {approximate }}-T_{\text {exact }}}{T_{\text {exact }}}\right|$,

where $T_{\text {exact }}$ is obtained from the parametric equation A-1. Within the layers above the reflector, we identify the layer with maximum horizontal velocity and mark its properties as $t_{0 M}, v_{h M}, \eta_{M}$, calculate the parameters that depend on the properties of all layers (equations 2 and 4), then calculate the parameters of each approximation.

All the studied approximations have three fitting constraints at zero offset; the three-parameter approximation of Alkhalifah and Tsvankin (1995) has no fitting constraint at infinity; the modified fourparameter approximation of Tsvankin and Thomsen (1994) has one fitting constraint at infinity, and our six-parameter approximation has three fitting constraints at infinity (Figure 2). Ravve and Koren (2017) use a different approach to obtain their equation parameters, which involves tracing one ray in lower velocity layers (explained in appendix A of Ravve and Koren, 2017). In equation B-14, we also provide a modified simpler parameter definition for their equation that results in two fitting constraints at infinity. As Figure 2 shows, our modified version of Ravve and Koren (2017) is more accurate than the original one. The value of our calculated $B_{L}$ (a parameter of Ravve and Koren (2017) equation) is different from the one obtained by Ravve and Koren (2017), which implies their method does not produce the second term of the exact asymptote accurately. With six -symmetrically distributed- fitting constraints at the two ends of a reflection traveltime, our proposed traveltime approximation is more accurate than the other approximations (Figure 2). 
Next, we consider a variety of layered VTI models. The models set consist of 1000 randomly generated models containing 2 to 14 VTI layers. The layer properties are selected within the predefined range $v_{P 0} \in[2,5] \mathrm{km} / \mathrm{s}, \eta \in[0,0.5], \delta \in[-0.1,0.1], \quad z \in[0.1,0.25] \mathrm{km}$. Figure 3 compares the maximum value of relative traveltime errors in the aforementioned set of models from zero to infinite offsets. In $99 \%$ of the models, the maximum error of our traveltime approximation (equation 5) is below 1\%. In this experiment, we could not find any specific model characteristic that results in errors above $1 \%$ in our approximation.

\section{APPLICATIONS}

\section{Forward modeling}

For modeling reflection events over a layered VTI model, we calculate the traveltime and amplitude of each reflection at the given source-to-receiver offsets. Equation 5 approximates the traveltimes as a function of offset, given a stratified earth model. There also exist equations for reflection and transmission coefficients at each interface (Graebner, 1992; Stovas and Ursin, 2003), but as a function of ray parameter $p$. Therefore, for direct analytical calculation of reflection amplitudes at given offsets, we need an equation of ray parameter as a function of offset. Taking the derivative of equation 5 with respect to offset, we obtain,

$$
p(X) \simeq \frac{X}{2 T V_{n}^{4}}\left(2 V_{n}^{2}+\frac{4 A X^{2}}{R_{1}+R_{2}}-\frac{2 A X^{4}}{\left(R_{1}+R_{2}\right)^{2}}\left(\frac{B+C X^{2}}{R_{1}}+\frac{D}{2 R_{2}}\right)\right),
$$

where $T(X)$ is the traveltime approximation in equation $5, \quad R_{1}=\sqrt{T_{0}^{4}+2 B X^{2}+C X^{4}}$ and $R_{2}=\sqrt{T_{0}^{4}+D X^{2}}$. Equation 10 approximates the ray parameter of each reflected ray traveling from a source to a receiver separated by offset $X$.

Figure 4a shows the earth model in Table 1 with reflected rays, traced for a large-offset common midpoint (CMP) acquisition pattern. Figure $4 \mathrm{~b}$ shows the relative errors of the approximated traveltime and ray parameters using equations 5 and 10, respectively. The ray parameter errors are generally higher than the traveltime errors due to the derivative effect in equation 10 .

Figure 5a shows the generated synthetic data based on exact ray tracing, and Figure $5 \mathrm{~b}$ shows the data based on our approximate forward modeling that calculates traveltimes with equation 5 , and amplitudes by inserting equation 10 into the Graebner (1992) equations. Despite the approximate nature, it is a fast analytical forward modeling approach. Figure $5 \mathrm{c}$ shows the difference between the exact and approximated data. The maximum difference in Figure $5 \mathrm{c}$ (around $x=0.5 \mathrm{~km}$ ) is the result of an overlap of the higher errors of the approximated $p$ and the critical reflection angle that results in a sharp change of reflection coefficient.

\section{Parameter estimation beneath a high-velocity layer}

Equation 5 is a six-parameter traveltime approximation. The higher the number of unknowns in model parameter estimations, the higher the uncertainty in inversion. To reduce the number of unknowns estimated from a reflection traveltime, we assume that the properties of the layer with maximum horizontal velocity in the sequence are known. An earth model with a shallow high-velocity layer overlaying deeper lower velocity layers is a case where we may obtain the properties of the high-velocity layer from conventional methods. When this known high-velocity layer has the maximum horizontal velocity in the sequence, we 
use equation 5 to estimate the four effective parameters $T_{0}, V_{n}, S_{2}$, and $S_{\infty}$ from reflections beneath it. Figure 6a shows a large-offset model with a high-velocity layer as the shallowest layer. Properties of each layer are presented in Figure 6 and 7. We find the best fit of equation 5 to the ray-traced traveltimes (using trust-region-reflective nonlinear least-squares curve fitting). For comparison, we use the four-parameter traveltime approximation of Tsvankin and Thomsen (1994), so that in the curve-fitting process both equations have four unknowns (degrees of freedom). Figure 6b shows the maximum relative errors of the best fit of the equations. Notice the high errors exhibited by the Tsvankin and Thomsen (1994) approximation in the reflections immediately after the high-velocity layer, and the improved accuracy in our approximation.

Figure $6 \mathrm{c}-6 \mathrm{e}$ shows the estimated parameters alongside their analytical values calculated from the model parameters using equations 2 and 4 . Figure $6 \mathrm{c}$ shows the estimated $V_{n}$, which is analytically associated with the second-order derivative of traveltime at zero offset; our new approach has estimated it with high accuracy. Figure 6d shows the conventional heterogeneity parameter $S_{2}$, which is analytically associated with the fourth-order derivative of traveltime at zero offset; our result is more accurate than that of Tsvankin and Thomsen (1994), although both equations have failed to estimate this parameter with high accuracy. Figure 6e shows our new heterogeneity parameter $S_{\infty}$ estimated with great accuracy by equation 5 . Comparing the values of $S_{2}$ and $S_{\infty}$, our new $S_{\infty}$ parameter presents a better indicator of the layering effect on traveltime because it strictly increases with the increase in the number of layers.

The estimated parameters can theoretically be converted to interval parameters of each layer (Dix, 1955; Tsvankin and Thomsen, 1994). Using a Dix-type differentiation, we calculate interval $v_{h}$ in each layer beneath the high velocity layer. From the difference between two subsequent estimations of $S_{\infty}$ at the top and bottom boundaries of the layer, we have:

$$
\left(S_{\infty(j)}-S_{\infty(j-1)}\right)^{2}=\frac{t_{0(j)}^{2}\left(v_{h M}^{2}-v_{h(j)}^{2}\right)}{t_{0 M}^{2}\left(v_{h M}^{2}-v_{h(j)}^{2}+v_{n(j)}^{2}\right)},
$$

where $j$ is the reflector number, $S_{\infty(j)}$ is the summation in equation 4 over $j$ layers, and $v_{h(j)}, t_{0(j)}$ and $v_{n(j)}$ are the properties of the $j$-th layer, which is the layer immediately above the reflector. Since in this application the layer with the maximum horizontal velocity is known, we can obtain $v_{h}$ of a layer beneath it by solving equation 11 :

$v_{h(j)}^{2}=v_{h M}^{2}+\frac{\left(S_{\infty(j)}-S_{\infty(j-1)}\right)^{2} t_{0 M}^{2} v_{n(j)}^{2}}{\left(S_{\infty(j)}-S_{\infty(j-1)}\right)^{2} t_{0 M}^{2}-t_{0(j)}^{2}}$,

Figure 7 shows the calculated interval parameters from the estimated effective parameters by our traveltime approximation that are presented in Figure 6. Here, the estimated interval properties match the model parameters appropriately. Nonetheless, this process inherently augments the errors in the estimated effective parameters because it involves differentiation. 


\section{DISCUSSION}

The calculated series in equation 3 provides insights into the traveltime behavior at infinitely large offsets in horizontally layered VTI media: it is dominated by the properties of the layer with the highest horizontal velocity (marked by subscript $M$ ). The leading term of the asymptotic series solely depends on $v_{h M}$. Calculating the relative traveltime error at infinity by inserting our calculated asymptote into equation 9 , an $a$ percent error in $v_{h M}$ results in a $|100 a /(100+a)|$ percent error in the traveltime at infinity. The effect of other layers, including the difference between their horizontal velocities and $v_{h M}$, appears in the series in a cumulative term that we separated as $S_{\infty}$. In practice, a refraction study can help estimate the horizontal velocities.

An important aspect of a traveltime approximation is the number of independent parameters it employs. These parameters are generally associated with different orders of derivatives of traveltime at specific offsets. The equations of Tsvankin and Thomsen (1994), Fomel and Stovas (2010), and Abedi and Stovas (2019) use four, five, and six parameters, respectively; but in layered media only one of their parameters can be found from a constraint at infinity (Appendix B). The Ravve and Koren (2017) approximation has five parameters; we found two of them from constraints at infinity (equation B-14), improving the Ravve and Koren (2017) original method that does not reproduce the second term of the asymptote. Our proposed approximation has six parameters symmetrically found from constraints at zero and infinite offsets.

A limitation of the proposed traveltime approximation is that in specific models in which some layers have negative anellipticity, some of its constraints at infinity will be lost. Although equation 8 mitigates this problem, it is not suitable for the estimation of model parameters based on a coherency measurement.

\section{CONCLUSIONS}

In layered VTI media, the traditional traveltime approximations that are based on Taylor-series expansion at zero offset become inaccurate at larger offsets. To address this issue, we first calculated the asymptotic series of the exact traveltime around infinite offset. Then, we constructed a new traveltime approximation that is accurate at both near and far offsets. Using the calculated series in the definition of our traveltime approximation parameters, our developed equation is explicitly defined from model properties without ray tracing. Numerical results show the superior accuracy of the proposed approximation compared to existing ones. In $99 \%$ of the studied models, the maximum error of our approximation is below $1 \%$. The proposed equation approximates reflection traveltimes, given the earth model properties. Therefore, it can be used for direct analytical forward modeling, as illustrated. The parameters of the proposed equation are the conventional effective zero offset traveltime, NMO velocity, and anellipticity, in addition to a new heterogeneity parameter and the properties of the layer with maximum horizontal velocity in the sequence. We showed how we can estimate the first four parameters from reflections beneath a known high-velocity layer. The new large-offset heterogeneity parameter strictly increases with increasing the number of layers, therefore, it also indicates the layering effect.

\section{ACKNOWLEDGEMENTS}

We are grateful to the journal reviewers A. Stovas, and two anonymous reviewers, and to the associate editor T. Alkhalifah and the editor in chief J. Shragge for their insightful comments and suggestions. This work is funded by the European Union's Horizon 2020 research and innovation program grant agreement No 777778 (MATHROCKS); the European Regional Development Fund (ERDF) through the Interreg V- 
A Spain-France-Andorra program POCTEFA 2014-2020 Project PIXIL (EFA362/19); the Spanish Ministry of Science and Innovation with references PID2019-108111RB-I00 (FEDER/AEI) and the "BCAM Severo Ochoa" accreditation of excellence (SEV-2017-0718); and the Basque Government through the BERC 2018-2021 program, the two Elkartek projects 3KIA (KK-2020/00049) and MATHEO (KK-2019-00085), the grant "Artificial Intelligence in BCAM number EXP. 2019/00432", and the Consolidated Research Group MATHMODE (IT1294-19) given by the Department of Education.

\section{APPENDIX A}

\section{DERIVATION OF THE ASYMPTOTIC SERIES AT INFINITY}

Equations of exact traveltime $(T)$ and offset $(X)$ over a $k$-layer acoustic VTI model exists as a function of ray parameter $(p)$ in a parametric form (rewritten from Fomel and Grechka, 2001),

$$
X(p)=\sum_{i=1}^{k} u_{x(i)^{w}}{ }_{(i)}, T(p)=\sum_{i=1}^{k} u_{t(i)} w_{(i)},
$$

where,

$$
\begin{aligned}
& u_{x(i)}=p v_{n(i)}^{2}, \\
& u_{t(i)}=\left(1-2 \eta_{(i)} p^{2} v_{n(i)}^{2}\right)^{2}+2 \eta_{(i)} p^{4} v_{n(i)}^{4}, \\
& w_{(i)}=t_{0(i)} / \sqrt{\left(1-2 \eta_{(i)} p^{2} v_{n(i)}^{2}\right)^{3}\left(1-p^{2} v_{h(i)}^{2}\right)} .
\end{aligned}
$$

The maximum possible horizontal slowness for a reflection in layered models is the inverse of $v_{h M}$, where $v_{h M}$ is the highest horizontal velocity in the sequence, and $M$ is the index of this layer. When $p \rightarrow 1 / v_{h M}$ , the term $w_{(M)}$ tends to infinity, therefore, the limits of equations A-1 also approach infinity:

$$
\begin{aligned}
& \lim _{p \rightarrow 1 / v_{h M}} T=\lim _{p \rightarrow 1 / v_{h M}}\left(u_{t(M)^{w_{(M)}}}+\sum_{i \neq M} u_{t(i)^{w_{(i)}}}\right), \\
& \lim _{p \rightarrow 1 / v_{h M}} X=\lim _{p \rightarrow 1 / v_{h M}}\left(u_{x(M)^{w_{(M)}}}+\sum_{i \neq M} u_{x(i)^{w_{(i)}}}\right) .
\end{aligned}
$$

In each of the above limits, the second term is finite (because $p<1 / v_{h(i)}$ ). Calculating the above limit for $T^{2}$, and neglecting the finite terms, we obtain:

$$
\lim _{p \rightarrow 1 / v_{h M}} T^{2}=\lim _{p \rightarrow 1 / v_{h M}}\left(u_{t(M)^{W}} w_{(M)}\right)^{2} \simeq \lim _{p \rightarrow 1 / v_{h M}}\left(\frac{u_{t(M)}^{2}}{u_{x(M)}^{2}} X^{2}\right) .
$$

From equation A-4, the leading term in the asymptotic approximation of traveltime squared is obtained as,

$T^{2} \approx c_{2} X^{2}$

where, 
$c_{2}=\left(u_{t(M)}^{2} u_{x(M)}^{-2}\right)_{p=1 / v_{h M}}$.

To obtain the second term of the asymptotic series, we repeat the aforementioned process for traveltime squared minus the first term:

$\lim _{p \rightarrow 1 / v_{h M}}\left(T^{2}-c_{2} X^{2}\right)=\lim _{p \rightarrow 1 / v_{h M}}\left(\begin{array}{l}\left\{u_{t(M)^{2}}^{2} w_{(M)}^{2}+\left(\sum_{i \neq M} u_{t(i)} w_{(i)}\right)^{2}+2 u_{t(M)} w_{(M)} \sum_{i \neq M} u_{t(i)} w_{(i)}\right\} \\ -c_{2}\left\{u_{x(M)^{w}}^{2} w_{(M)}^{2}+\left(\sum_{i \neq M} u_{x(i)} w_{(i)}\right)^{2}+2 u_{x(M)} w_{(M)} \sum_{i \neq M} u_{x(i)} w_{(i)}\right\}\end{array}\right\}$.

In a homogeneous model, the summations in equation A-7 become empty. Therefore, equation A-7 becomes finite,

$\lim _{p \rightarrow 1 / v_{h M}}\left(T^{2}-c_{2} X^{2}\right)=\lim _{p \rightarrow 1 / v_{h M}}\left(\left\{c_{2} u_{x(M)}^{2}-u_{t(M)}^{2}\right) w_{(M)}^{2}\right)$.

Otherwise, when equation A-7 is infinite, neglecting the finite terms in it,

$$
\begin{aligned}
\lim _{p \rightarrow 1 / v_{h M}}\left(T^{2}-c_{2} X^{2}\right) & =\lim _{p \rightarrow 1 / v_{h M}}\left(2 u_{t(M)^{w}} w_{(M)}\left\{\sum_{i \neq M} u_{t(i)} w_{(i)}-\frac{u_{t(M)}}{u_{x(M)}} \sum_{i \neq M} u_{x(i)} w_{(i)}\right\}\right) \\
& \simeq \lim _{p \rightarrow 1 / v_{h M}}\left(2 u_{t(M)} u_{x(M)}^{-2}\left\{u_{x(M)} \sum_{i \neq M} u_{t(i)} w_{(i)}-u_{t(M)} \sum_{i \neq M} u_{x(i)} w_{(i)}\right\} X\right) .
\end{aligned}
$$

From the above equation, we add the second term in the asymptotic approximation of traveltime squared as,

$T^{2} \approx c_{2} X^{2}+c_{1} X$

where,

$c_{1}=\left(2 u_{t(M)} u_{x(M)}^{-2}\left\{u_{x(M)} \sum_{i \neq M} u_{t(i)^{w}{ }_{(i)}}-u_{t(M)} \sum_{i \neq M} u_{x(i)} w_{(i)}\right\}\right)_{p=1 / v_{h M}}$.

To continue with the third term of the asymptotic series, we repeat a similar process for traveltime squared minus the already calculated terms:

$$
\lim _{p \rightarrow 1 / v_{h M}}\left(T^{2}-c_{2} X^{2}-c_{1} X\right)=\lim _{p \rightarrow 1 / v_{h M}}\left(\begin{array}{l}
\left\{\left(\sum_{i \neq M} u_{t(i)} w_{(i)}\right)^{2}-c_{1} \sum_{i \neq M} u_{x(i)} w_{(i)}-c_{2}\left(\sum_{i \neq M} u_{x(i)} w_{(i)}\right)^{2}\right\} \\
+\left\{2 u_{t(M)} \sum_{i \neq M} u_{t(i)} w_{(i)}-2 c_{2} u_{x(M)} \sum_{i \neq M} u_{x(i)} w_{(i)}-c_{1} u_{x(M)}\right\} w_{(M)} \\
+\left\{c_{2} u_{x(M)}^{2}-u_{t(M)}^{2}\right\} w_{(M)}^{2}
\end{array}\right),
$$

If we neglect the finite terms in equation A-12, it will equal zero, therefore, the third term is finite. Adding the third term, the asymptotic approximation of traveltime squared reads, 
$T^{2}(X \rightarrow \infty) \approx c_{2} X^{2}+c_{1} X+c_{0}$,

where,

$$
\begin{aligned}
c_{0} & =\left(u_{x(M)}^{-2}\left(u_{t(M)} \sum_{i \neq M} u_{x(i)} w_{(i)}-u_{x(M)} \sum_{i \neq M} u_{t(i)} w_{(i)}\right)^{2}\right)_{p=1 / v_{h M}} \\
& +\lim _{p \rightarrow 1 / v_{h M}}\left(\left\{c_{2} u_{x(M)}^{2}-u_{t(M)}^{2}\right\} w_{(M)}^{2}\right),
\end{aligned}
$$

is obtained after removing the part of equation A-12 that tends to zero. We repeat this recursive process to obtain more terms of the asymptotic series. The terms of the series have the form $c_{m} X^{m}$ with coefficients,

$c_{m}=\lim _{p \rightarrow 1 / v_{h M}} \frac{T^{2}(p)-\sum_{k=m, k \leq 1}^{2} c_{k+1} X^{k+1}(p)}{X^{m}(p)}$,

where $m \leq 2$ and includes negative integers. The calculated asymptotic series in equation A-15 with simplified coefficients is presented in equation 3. In equations A-11 and A-14, we find a common term, which forms our $S_{\infty}$ after normalization by $t_{0 M}$ :

$S_{\infty}=\left(t_{0 M}^{-1} u_{x(M)}^{-1}\left\{u_{x(M)} \sum_{i \neq M} u_{t(i)^{W}{ }_{(i)}}-u_{t(M)} \sum_{i \neq M} u_{x(i)} w_{(i)}\right\}\right)_{p=1 / v_{h M}}$.

In a homogeneous medium, the summations in the above equation equal zero (because $i \neq M$ is nil), resulting in $S_{\infty}=0$.

\section{APPENDIX B}

\section{PARAMETER DEFINITION FOR TRAVELTIME APPROXIMATIONS}

To define the parameters of a traveltime approximation as a function of the model properties, we equate the terms of the series of the approximate equation to the corresponding terms of the series of the exact traveltime. This appendix shows this process for several traveltime approximations. The traveltime approximation by Abedi and Stovas (2019b) reads,

$$
T^{2}(X) \simeq T_{0}^{2}+\frac{X^{2}}{V_{n}^{2}}+\frac{A X^{4}}{V_{n}^{4}\left(T_{0}^{2}+D \frac{X^{2}}{V_{n}^{2}}+\sqrt{T_{0}^{4}+2 B \frac{T_{0}^{2} X^{2}}{V_{n}^{2}}+C \frac{X^{4}}{V_{n}^{4}}}\right)} .
$$

Equation B-1 is reduced to the Fomel and Stovas (2010) equation when setting $D=B$, and to Tsvankin and Thomsen (1994) equation when setting $C=B^{2}$, and $D=B$. The Ravve and Koren (2017; equation 57) approximation reads, 


$$
T^{2}(X) \simeq T_{0}^{2}+\frac{X^{2}}{V_{n}^{2}}+\frac{A X^{4}}{V_{n}^{4}\left(B_{H} \frac{X^{2}}{V_{n}^{2}}+\sqrt{T_{0}^{4}+2 B_{L} \frac{T_{0}^{2} X^{2}}{V_{n}^{2}}}\right)} .
$$

In the aforementioned traveltime approximations, $T_{0}, V_{n}, A$, are defined at zero offset, and $B, C, D$ or $B_{H}$ and $B_{L}$ are parameters that we try to find for each approximation at infinity.

At zero offset, the Taylor-series expansion of the Fomel and Stovas (2010), Abedi and Stovas (2019b), and our approximation in equation 5 are identical up to the third term,

$T^{2}(X \rightarrow 0) \approx T_{0}^{2}+\frac{X^{2}}{V_{n}^{2}}+\frac{A X^{4}}{2 T_{0}^{2} V_{n}^{4}}+\ldots$

The Taylor-series expansions of the Tsvankin and Thomsen (1994) and Ravve and Koren (2017) approximations are also similar to the series in equation B-3, but without the factor of 2 in the third term.

At infinite offsets, we calculate the asymptotic series of each equation, using a similar method to that in Appendix A. The asymptote has the form

$T^{2}(X \rightarrow \infty) \approx d_{2} X^{2}+d_{1} X+d_{0}+\ldots$

with coefficients,

$d_{2}=\lim _{X \rightarrow \infty} \frac{T^{2}}{X^{2}}$

$d_{1}=\lim _{X \rightarrow \infty} \frac{T^{2}-d_{2} X^{2}}{X}$,

$d_{0}=\lim _{X \rightarrow \infty}\left\{T^{2}-d_{2} X^{2}-d_{1} X\right\}$.

Note that the traveltime approximations are explicit functions of $X$.

At infinite offset, we calculate the asymptotic series of the approximations from Tsvankin and Thomsen (1994),

$T^{2}(X \rightarrow \infty) \approx \frac{A+B}{B V_{n}^{2}} X^{2}+\left(1-\frac{A}{B^{2}}\right) T_{0}^{2}+\ldots$

Fomel and Stovas (2010),

$T^{2}(X \rightarrow \infty) \approx \frac{A+B+\sqrt{C}}{(B+\sqrt{C}) V_{n}^{2}} X^{2}+\frac{C-A+B \sqrt{C}}{B \sqrt{C}+C} T_{0}^{2}+\ldots$,

Abedi and Stovas (2019b),

$T^{2}(X \rightarrow \infty) \approx \frac{A+D+\sqrt{C}}{(D+\sqrt{C}) V_{n}^{2}} X^{2}+\left(1-\frac{A(B+\sqrt{C})}{\sqrt{C}(\sqrt{C}+D)^{2}}\right) T_{0}^{2}+\ldots$, 
Ravve and Koren (2017; equation 57),

$T^{2}(X \rightarrow \infty) \approx \frac{A+B_{H}}{B_{H} V_{n}^{2}} X^{2}-\frac{A \sqrt{B_{L}} T_{0}}{B_{H}^{2} V_{n}} X+\left(1+\frac{A B_{L}}{B_{H}^{3}}\right) T_{0}^{2}+\ldots$,

and our approximation in equation 5,

$T^{2}(X \rightarrow \infty) \approx\left(\frac{A}{V_{n}^{4} \sqrt{C}}+\frac{1}{V_{n}^{2}}\right) X^{2}-\frac{A \sqrt{D}}{C V_{n}^{4}} X+\left(T_{0}^{2}-\frac{A(B-D)}{V_{n}^{4} \sqrt{C^{3}}}\right)+\ldots$

We equate different terms of the above series to the corresponding terms of the series of exact traveltime (equation 3) to find the parameters of each equation in layered VTI media. Equations B-6 - B-8 do not include the odd terms of $X$, therefore, only the first term of their asymptote at infinity can be matched to equation 3. From equation B-6 we obtain,

$B=-\frac{A v_{h M}^{2}}{2\left(v_{h M}^{2}-V_{n}^{2}\right)}$,

and from equations B-7 and B-8, we obtain,

$C=\frac{\left(A v_{h M}^{2}+B\left(v_{h M}^{2}-V_{n}^{2}\right)\right)^{2}}{\left(v_{h M}^{2}-V_{n}^{2}\right)^{2}}$

$C=\frac{\left(A v_{h M}^{2}+D\left(v_{h M}^{2}-V_{n}^{2}\right)\right)^{2}}{\left(v_{h M}^{2}-V_{n}^{2}\right)^{2}}$

respectively, but we cannot find the parameters $B$ (and $D$ ) of the Fomel and Stovas (2010) and Abedi and Stovas (2019b) from the asymptotic fit at infinite offsets in layered media. $B$ (and $D$ ) in these approximations may be found by employing more fitting constraints at zero offset, but the asymptotic accuracy of Fomel and Stovas (2010), Abedi and Stovas (2019b), and Tsvankin and Thomsen (1994) will be the same. From equation B-9, we obtain,

$B_{H}=-\frac{A v_{h M}^{2}}{\left(v_{h M}^{2}-V_{n}^{2}\right)}, B_{L}=\frac{4 A^{2} S_{\infty}^{2} t_{0 M}^{2} v_{h M}^{6} V_{n}^{2}}{T_{0}^{2}\left(v_{h M}^{2}-V_{n}^{2}\right)^{4}}$.

The definition of $B_{H}$ is the same as the original definition by Ravve and Koren (2017), but the definition of $B_{L}$ in equation B-14 is a modified explicit definition of this parameter for the Ravve and Koren (2017) approximation in equation B-2.

Only the series of our approximation (equation B-10) can be matched to the three terms of the exact series in equation 3 because it has the correct powers of $X$ and three degrees of freedom in their coefficients $\left(T_{0}\right.$, $V_{n}$, and $A$ are already defined at zero offset). The result is in equation 7. 


\section{REFERENCES}

Abedi, M.M., 2020, Rational approximation of P-wave kinematics - Part 1: Transversely isotropic media: Geophysics, 85, no.5, C175-C185.

Abedi, M.M., and A. Stovas, 2019a, A new parameterization for generalized moveout approximation, based on three rays: Geophysical Prospecting, 67, no.5, 1243-1255. DOI:10.1111/1365-2478.12770

Abedi, M.M., and A. Stovas, 2019b, Extended generalized nonhyperbolic moveout approximation: Geophysical Journal International, 216, 1428-1440.

Abedi M.M., M.A. Riahi, and A. Stovas, 2019a, Three-parameter NMO correction in layered anisotropic media: a stretch-free approach: Geophysics, 84, no. 3, C129-C142.

Abedi M.M., M.A. Riahi, and A. Stovas, 2019b, Three-parameter Radon transform in layered transversely isotropic media: Geophysical Prospecting, 67, 395-407.

Alkhalifah, T., 1997, Velocity analysis using nonhyperbolic moveout in transversely isotropic media: Geophysics, 62, 1839-1854.

Alkhalifah, T., 1998, Acoustic approximations for processing in transversely isotropic media: Geophysics, 63, 623-631.

Alkhalifah T., 2011, Scanning anisotropy parameters in complex media: Geophysics 76, no. 2, U13-22.

Alkhalifah T., and I. Tsvankin, 1995, Velocity analysis for transversely isotropic media: Geophysics, 60, 1550-1566.

Causse, E., G.U. Haugen, and B.E., Rommel, 2001, Large-offset approximation to seismic reflection traveltimes: Geophysical Prospecting, 48, no. 4, 763-778.

Colombo, D., 2005, Benefits of wide-offset seismic for commercial exploration targets and implications for data analysis: The Leading Edge, 24, no. 4, 352-363.

Dai, H. and X.Y., Li, 2001, Anisotropic migration and model building for 4C seismic data: A case study from Alba: Annual International Meeting, SEG, Expanded Abstracts 795-798.

de Bazelaire, E., 1988, Normal moveout revisited-Inhomogeneous media and curved interfaces: Geophysics, 53, 142-58.

Dix, C.H., 1955. Seismic velocities from surface measurements. Geophysics, 20, no.1, 68-86.

Farra, V. and I., Pšenčík, 2020, P-wave reflection-moveout approximation for horizontally layered media of arbitrary moderate anisotropy: Geophysics, 85, no.2, C61-C70.

Fomel S., 2004, On anelliptic approximations for qP velocities in VTI media: Geophysical Prospecting, 52, no.3, 247-259.

Fomel, S. and V., Grechka, 2001, Nonhyperbolic reflection moveout of P waves. An overview and comparison of reasons. CWP-372: Colorado School of Mines.

Fomel, S., and A. Stovas, 2010, Generalized nonhyperbolic moveout approximation: Geophysics, 75, no. 2, U9-U18.

Graebner, M., 1992, Plane-wave reflection and transmission coefficients for a transversely isotropic solid: Geophysics, 57, 1512-1519. 
Hake, H., K. Helbig, and C.S., Mesdag, 1984, Three-term Taylor series for t2-x2-curves of P-and S-waves over layered transversely isotropic ground: Geophysical Prospecting, 32, no. 5, 828-850.

Ibanez-Jacome, W., T. Alkhalifah, and U.B. Waheed, 2014, Effective orthorhombic anisotropic models for wavefield extrapolation: Geophysical Journal International, 198 no.3, 1653-1661.

Muir F., and J. Dellinger, 1985, A practical anisotropic system: SEP 44, 55-58. Stanford Exploration Project.

Ravve I., and Z. Koren, 2017, Traveltime approximation in vertical transversely isotropic layered media: Geophysical Prospecting, 65, no.6, 1559-1581.

Stovas, A. and S., Fomel, 2017, The modified generalized moveout approximation: a new parameter selection: Geophysical Prospecting, 65, no. 3, 687-695.

Stovas, A. and B., Ursin, 2003, Reflection and transmission responses of layered transversely isotropic viscoelastic media: Geophysical Prospecting, 51, no.5, 447-477.

Taner, M. T., S. Treitel, and M. Al-Chalabi, 2005, A new travel time estimation method for horizontal strata: Annual International Meeting, SEG, Expanded Abstracts, 2273-2276.

Thomsen, L., 1986, Weak elastic anisotropy: Geophysics, 51, 1954-1966.

Tsvankin I., and L. Thomsen, 1994, Nonhyperbolic reflection moveout in anisotropic media: Geophysics, 59, 1290-1304.

Ursin B., and A. Stovas, 2006, Traveltime approximations for a layered transversely isotropic medium: Geophysics, 71, no.2, D23-33.

Table 1. Properties of the synthetic Model 1 used in generating Figures 1, 2, 4, and 5.

\begin{tabular}{c|cccccccc} 
Layer number & 1 & 2 & 3 & 4 & 5 & 6 & 7 & 8 \\
\hline$v_{P 0}(\mathrm{~km} / \mathrm{s})$ & 2.7 & 3.0 & 2.0 & 2.9 & 2.0 & 3.8 & 3.0 & 3.5 \\
$v_{n}(\mathrm{~km} / \mathrm{s})$ & 2.73 & 3.07 & 2.07 & 3.04 & 2.12 & 4.07 & 3.25 & 3.83 \\
$\eta$ & 0.09 & 0.49 & 0.27 & 0.16 & 0.29 & 0.18 & 0.44 & 0.07
\end{tabular}


a)

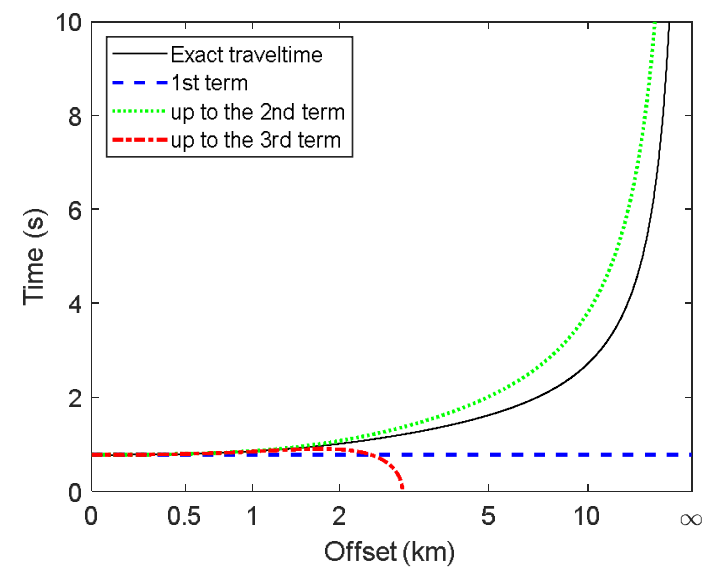

b)

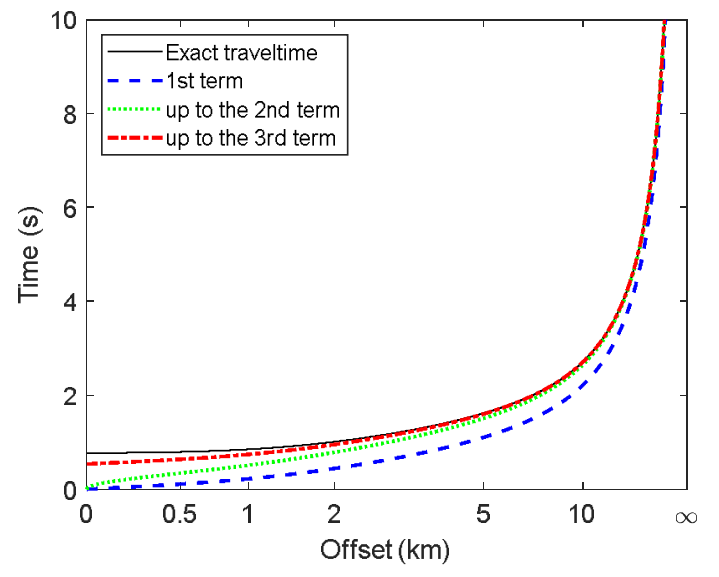

Figure 1. The exact traveltime in a layered VTI model (the fourth reflection in Table 1) and a) the series expansion around zero offset, b) the series expansion at infinity offsets. The horizontal axis is linear in phase angle.

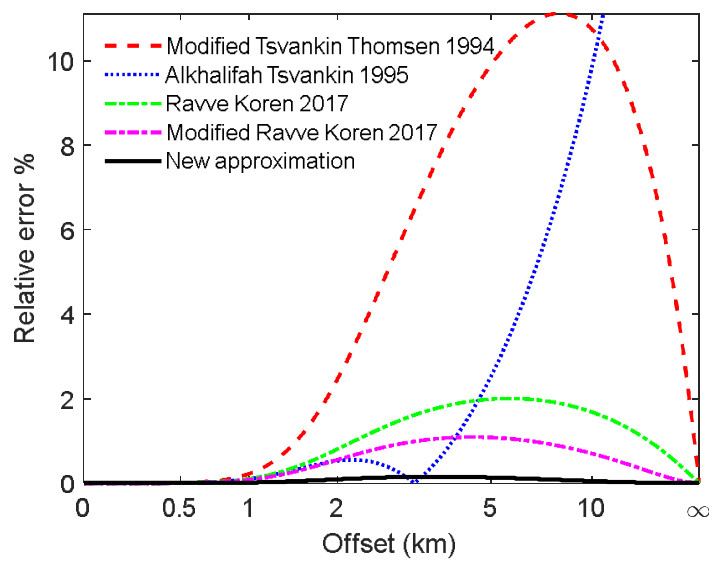

Figure 2. The absolute value of the relative error of the new traveltime approximation (equation 5) in a layered VTI model (the fourth reflection in Table 1), compared with other approximations. At infinity, the modified Tsvankin and Thomsen (1994) approximation has one fitting constraint (equation B-11), the modified Ravve and Koren (2017) has two fitting constraints (equation B-14), and our new approximation has three fitting constraints. 


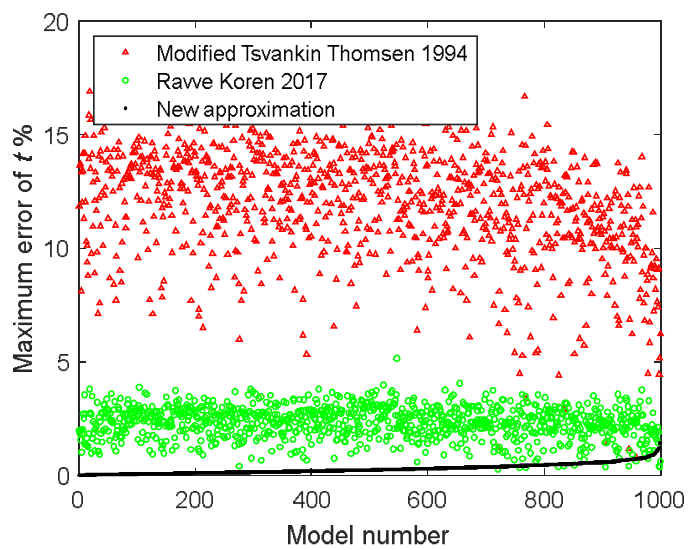

Figure 3. The maximum value of relative traveltime error of different approximations in a variety of randomly generated multilayer VTI models (up to infinite offset). The model numbers are sorted by the error values of the new approximation.

a)

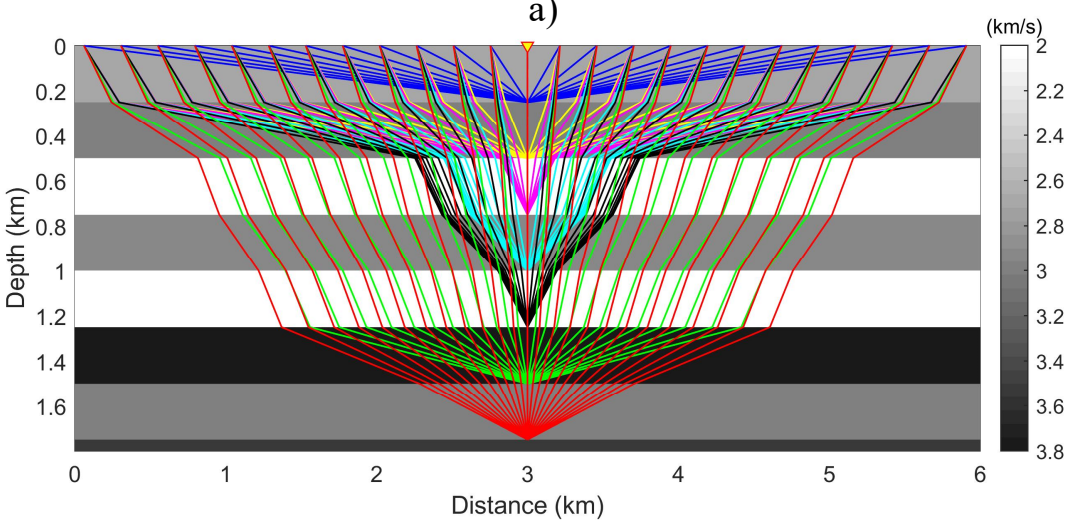

b)

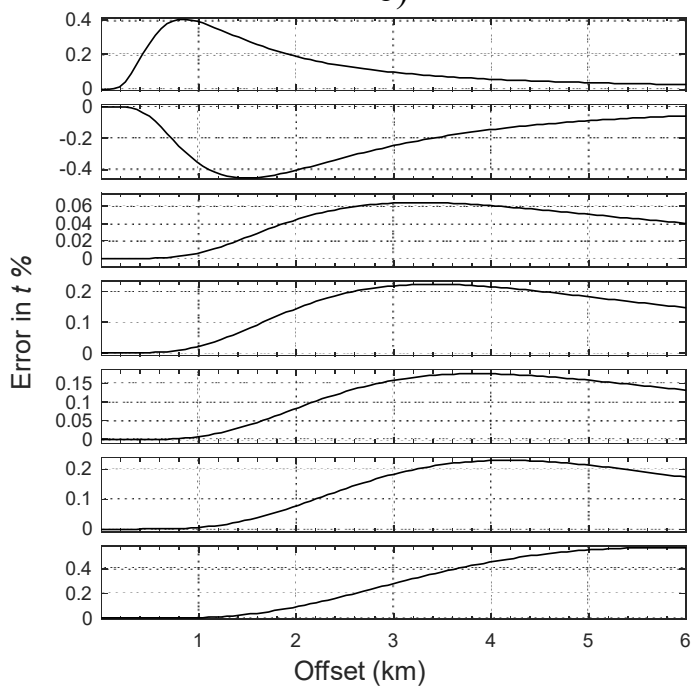

c)

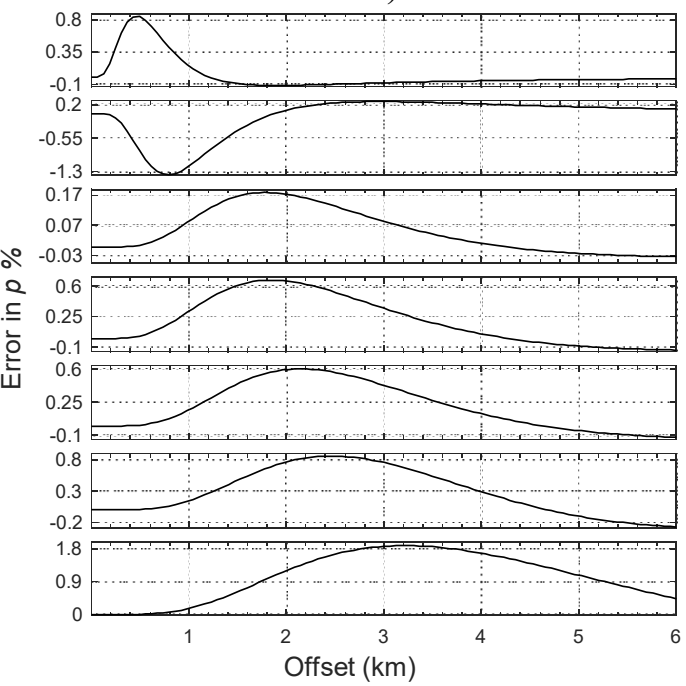

Figure 4. a) A large-offset model with representative traced rays in a CMP pattern. b) Relative errors of reflections traveltimes approximated with equation $5, \mathrm{c}$ ) relative errors of ray parameters approximated with equation 10 . 
a)

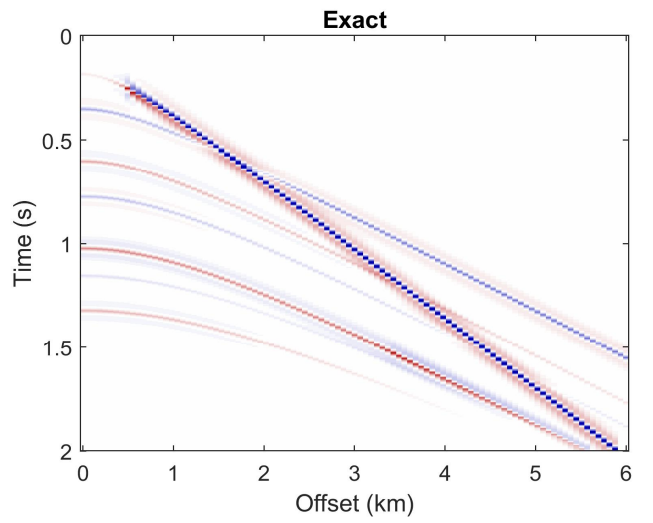

b)

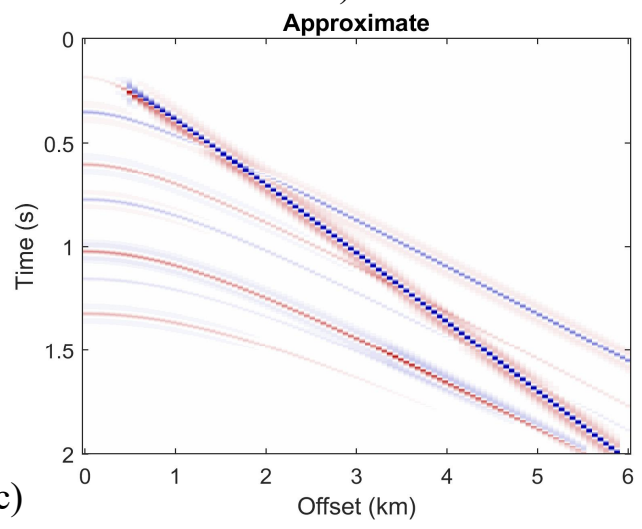

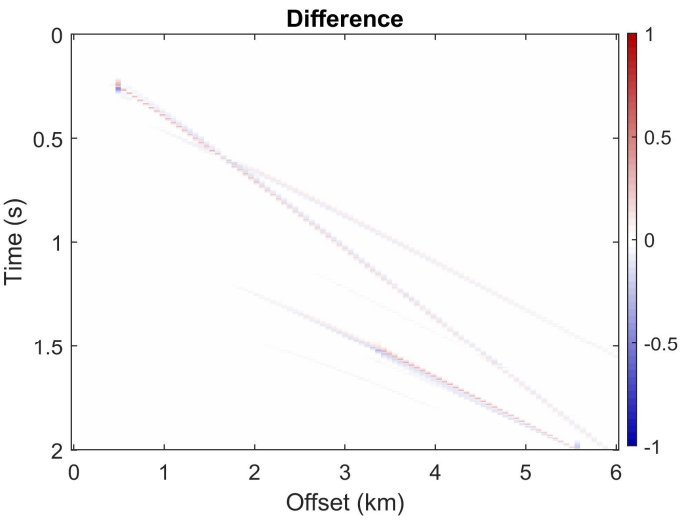

Figure 5. Synthetic data generated based on (a) the exact ray-tracing, and (b) the approximate traveltime and ray parameters in Figure 4. c) The difference between (a) and (b). 
a)

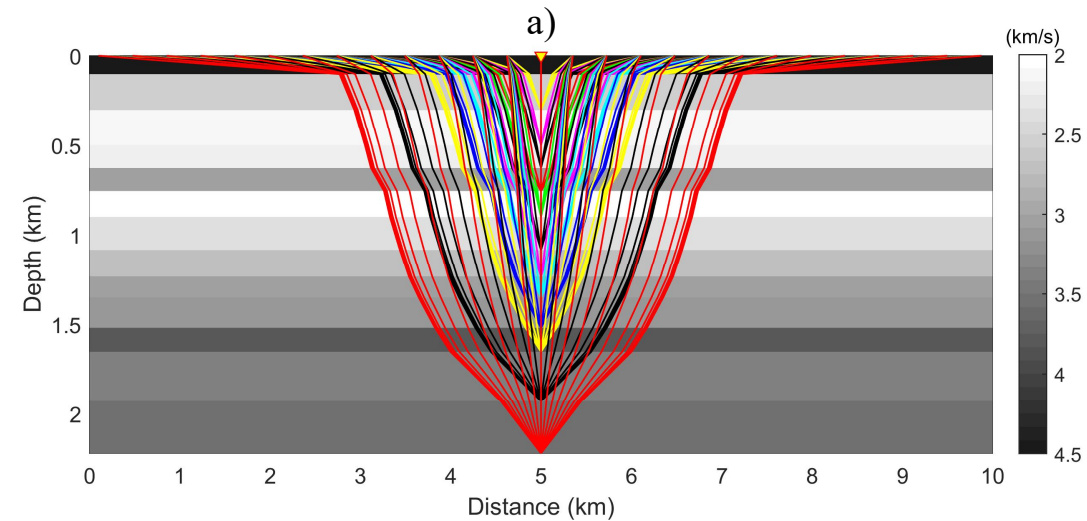

b)
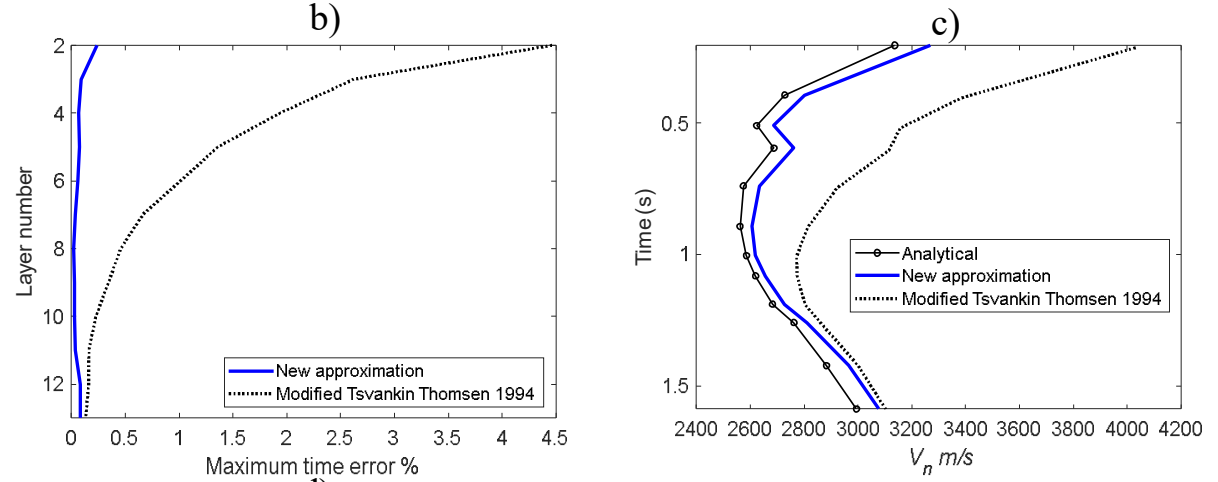

d)
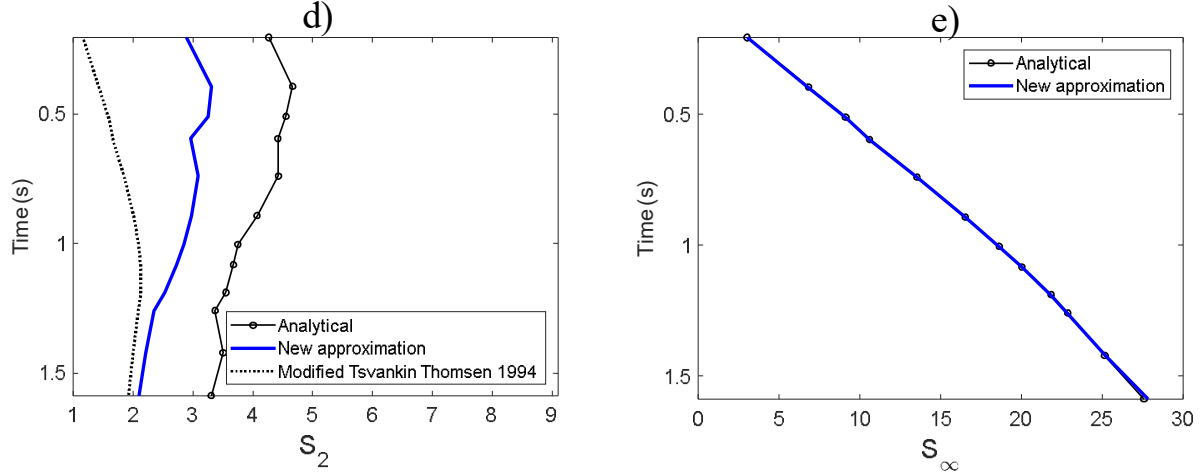

Figure 6. a) A large-offset model with a known high-velocity layer as the first layer. b) Relative errors of the best fit of traveltime approximations to the ray-traced traveltimes. c) Estimated effective NMO velocity, d) conventional heterogeneity, and e) the new large-offset heterogeneity parameter of equation 5. Analytical values, calculated based on the model parameters, are also shown. 
a)

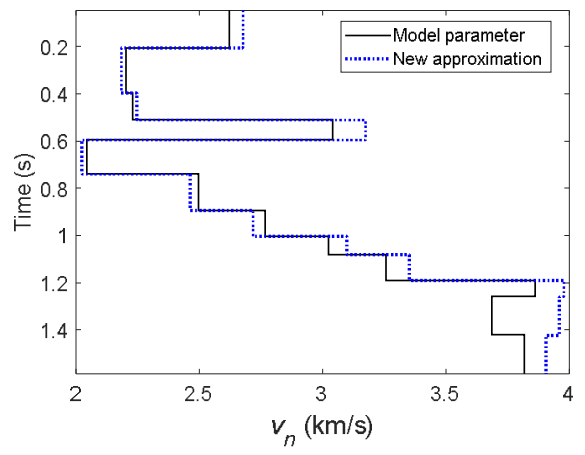

b)

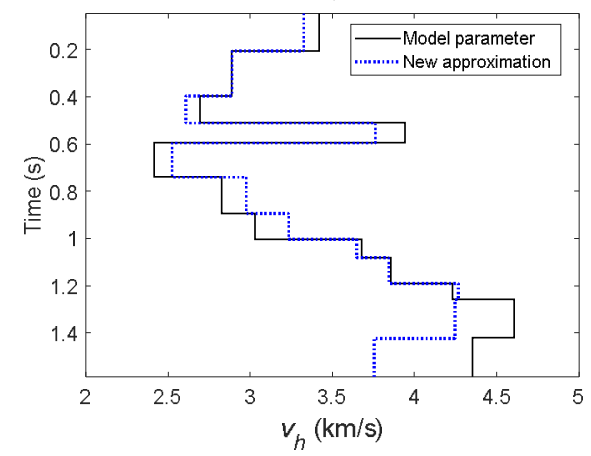

Figure 7. Interval NMO velocity (a), and horizontal velocity (b) of each layer, calculated from the estimated effective parameters in Figure 6, using equation 11. 